chapter on signalization emphasizes a formula by F. V. Webster for delays at isolated intersections, based on computer simulation of a queueing analogy. Two chapters on stochastic modelling begin with derivations of basic distributions and proceed, through the formalism for a single channel queue, to problems in overtaking, delays at intersections, merging and bottlenecks. After some discussion of simulation techniques, the book closes with a somewhat irrelevant, though fascinating, discussion of accident statisties with emphasis on determining accidentproneness among drivers.

The book is often turgid, uneven, and unnecessarily mysterious regarding foundations for the arguments used. For example, the stochastic material begins by assuming no previous knowledge, and there is even a derivation of the Poisson distribution, while the powerful PollaczekKhintchine formula is introduced without identification or discussion. In contrast, the treatment of stability in car following requires substantial external knowledge of advanced solution techniques for differential equations. A formula, for generating pseudo-random numbers alleged to follow "the" observed speed distribution, is offered without any hint that it samples an approximately normal distribution. There is also little awareness of the need for experimental verification, and thus Webster's intersection queueing simulation is "verified" by an inevitable agreement with a queueing analysis of a similar model by G. Newell. When confronted by three explanations of the difference between space and time average velocities, the reader will remain confused because of an erroneous characterization, as a time average, of the space average obtained from two successive photographs of a long road. There are too few diagrams; there is none to illustrate parameter measurement or the behaviour of shock waves in the fluid analogy.

In the absence of competition, this book is a marginally acceptable choice as a theory text for traffic engineers.

Walter Helly

\section{CHAINS OF PROBABILITY}

\section{Denumerable Markov Chains}

By John G. Kemeny, Laurie J. Snell and Anthony W. Knapp. Pp. $x i+439$. (The University Series in Higher Mathematics.) (Princeton, N.J.: D. Van Nostrand Company, Inc.; London: D. Van Nostrand Company, Ltd., 1966.) 100s.

The theory of denumerable Markov chains was set in motion by Kolmogorov in 1936, and its basic results have been accessible to students and research workers since the appearance of W. Feller's classical text on Probability Theory and its Applications in 1950. A more detailed treatment, covering progress up to about 1960 , is available in the monograph by K. L. Chung. The theory has been developing very rapidly, and there is certainly room for a new account which includes the most recent results. This the volume under review sets out to achieve. The treatment is almost self-contained, at a level suitable for research students rather than undergraduates, although the reader will find some familiarity with elementary probability theory helpful.

The book, as the authors explain, is in four parts. Part I provides background material, mainly dealing with the particular parts of probability theory needed for Markov chains, and the authors succeed rather well in discussing things like conditional probabilities and martingales clearly but without excessive generality. Part II deals with the basic theory of Markov chainsclassification of states and the limiting behaviour of transition probabilities. Parts III and IV go beyond anything to be found in existing monographs, because they deal first with discrote potential theory and its relation to Markov chain theory and then with the theory of boundaries. Essentially these are new aspects of the theory of Markov chains, uncovered within the last ten years by Feller, Doob and Hunt, and further developed by others including the first two of the three authors of this book. The treatment of boundary theory in Part IV will be particularly welcome to those who are hoping to learn about this important but rather difficult subject.

The book is well organized, though one may suspect that the elaborate system of special notations could be a hindrance to those readers who are looking for some particular result. I confess that I found the rather formal style to have too little variation of emphasis to hold the attention easily, but was certainly delighted to see such a lucid and coherent account of recent progress in Markov chains with an excellent list of references, including many papers published since 1960.

G. E. H. Reuter

\section{OLD ADAM'S LIKENESS}

\section{Man-Apes or Ape-Men?}

The Story of Discoveries in Africa. By Wilfrid E. Le Gros Clark. Pp. vii +150. (New York and London: Holt, Rinehart and Winston, Inc., 1967.) \$3.95.

STUDENTS of human palaeontology should be exceedingly grateful to Professor Le Gros Clark. In his new book he presents an admirably clear and concise account of the australopithecine discoveries in South and East Africa and of the scientific controversies that have bedevilled, befogged and, finally, illuminated the subject. That the author was in the very eye of most of these storms makes his account all the more piquant.

The book comprises a careful analysis of the comparative anatomy of the known remains of australopithecines at a level which will be acceptable to anatomists and non-anatomists alike: the illustrations are clear and helpful. The knowledge that the author has examined personally the majority of the bones discussed adds great weight to his conclusions.

Professor Le Gros Clark leaves no doubt as to where he stands in reference to various "bones of contention", notably those of Homo habilis, which he regards as "a geographical variant or just possibly a distinct local species (of Australopithecus)". In an interesting section dealing with behaviour, the author favours the view that Australopithecus was a toolmaker. This view can scarcely be upheld without the evidence provided by the pebbletool culture found on the Homo habilis living sites at Olduvai. Might it not be valid to consider that this hominid population were behaviourally more advanced than the broadly contemporary South African forms? The author has long contended that the definition of man will ultimately have to be based on some aspect of their behaviour. Is not cultural toolmaking still the most valid criterion to determine the threshold between "men" and "ape-men"?

J. R. NAPIER

\section{UNDERSTANDING ECONOMICS}

\section{The Economics of the Developing Countries}

By H. Myint. Third edition. (Hutchinson University Library.) Pp. 192. (London: Hutchinson and Co. (Publishers), Ltd., 1967.) 10s. 6d. paperbound; $25 s$. clothbound.

Export Instability and Economic Development

By Alasdair I. Macbean. (University of Glasgow Social and Economic Studies.) Pp. 364. (London: George Allen and Unwin, Ltd., 1966.) 45s. net.

Economics is a difficult subject. It is also becoming more and more unintelligible to anyone who is not highly specialized in the field. This is a pity, because obstacles 